\title{
Aspectos da territorialização do cuidado em um CAPSij: estudo seccional
}

\author{
Aspects of territorialization of care in a CAPSij: sectional study \\ Aspectos de la territorialización de la atención en un CAPSij: estudio seccional
}

Recebido: 28/07/2021 | Revisado: 03/08/2021 | Aceito: 04/08/2021 | Publicado: 08/08/2021

Luís Eduardo Batista

ORCID: https://orcid.org/0000-0003-4306-3426 Secretaria de Estado da Saúde de São Paulo, Brasil E-mail: lebatista@ saude.sp.gov.br

Ricardo Vicente Ferreira

ORCID: https://orcid.org/0000-0001-9003-4834 Universidade Federal do Triângulo Mineiro, Brasil E-mail: ricardo.ferreira@uftm.edu.br

Antônio Carlos Malachias

ORCID: https://orcid.org/0000-0003-2441-9431 Universidade de São Paulo, Brasil E-mail: billymalach@usp.br

Caroline Ballan

ORCID: https://orcid.org/0000-0002-8908-6669 Universidade de São Paulo, Brasil E-mail: caroline.ballan@usp.br

Sônia Barros

ORCID: https://orcid.org/0000-0002-6346-9717 Universidade de São Paulo, Brasil E-mail: sobarros@usp.br

Jussara Carvalho dos Santos

ORCID: https://orcid.org/0000-0002-7008-2756

Universidade de São Paulo, Brasil E-mail: jusantos@usp.br

\begin{abstract}
Resumo
Este estudo tem como objetivo integrar análises geográficas, sociais e de saúde considerando as variáveis raça/cor, para compreensão da complexidade da territorialização do cuidado em saúde mental. Para isto se propõe a responder à seguinte questão: quais as características sociodemográficas do território ocupado pelas crianças, mulheres e adolescentes na área de abrangência do CAPSij Brasilândia? Metodologia: Estudo seccional que permite análise e avaliação de riscos à saúde coletiva a que uma população está exposta, particularmente socioeconômicos e ambientais. O georreferenciamento descreve e permite a visualização da distribuição espacial, marcando determinantes sociais e fatores etiológicos que possam formular hipóteses e apontar associações entre os fenômenos. Com a tecnologia SIG buscou-se delimitar a área de abrangência do serviço, bem como, classificar aquilo que denominamos por complexidades para territorialização do cuidado em saúde mental. Resultados: A distribuição espacial das famílias (mulheres, crianças e adolescentes negros), acompanhadas pelo CAPSij mostra a segregação territorial vivenciada pela população pobre e negra. Os territórios ocupados pela maioria da população negra são retratos do racismo que estrutura a trama social brasileira. Conclusão: os territórios são produtos das relações sociais, podendo conferir vantagens e desvantagens ligadas a localização, infraestrutura, mobilidade, educação, trabalho, saúde, cultura, etc. Essa realidade configura os processos de saúde mental da população negra. Este estudo sobre a variação espacial no recorte raça/cor em serviços públicos de saúde possibilita visibilizar como as desigualdades raciais impactam no acesso ao direito à saúde, e apontar intervenções na perspectiva dos direitos humanos.
\end{abstract}

Palavras-chave: Território; Saúde mental; População negra; Direitos.

\begin{abstract}
This study aims to integrate geographic, social and health analyses considering the variables race/color, for understanding the complexity of territorialization of mental health care. For this, it proposes to answer the following question: what are the sociodemographic characteristics of the territory occupied by children, women and adolescents in the area covered by CAPSij Brasilândia? Methodology: Sectional study that allows analysis and evaluation of collective health risks to which a population is exposed, particularly socioeconomic and environmental risks. The georeferencing describes and allows the visualization of spatial distribution, marking social determinants and etiological factors that can formulate hypotheses and point out associations between the phenomena. With the GIS technology we sought to delimit the area of coverage of the service, as well as, to classify what we call by complexities for territorialization of mental health care. Results: The spatial distribution of families (black women,
\end{abstract}


children and adolescents), accompanied by CAPSij shows the territorial segregation experienced by the poor and black population. The territories occupied by the majority of the black population are portraits of the racism that structures the Brazilian social fabric. Conclusion: territories are products of social relations, and can confer advantages and disadvantages related to location, infrastructure, mobility, education, work, health, culture, etc. This reality shapes the mental health processes of the black population. This study on the spatial variation in the race/color clipping in public health services allows us to see how racial inequalities impact access to the right to health, and to point out interventions from the perspective of human rights.

Keywords: Territory; Mental health; Black population; Rights.

\section{Resumen}

Este estudio pretende integrar los análisis geográficos, sociales y sanitarios considerando las variables raza/color, para comprender la complejidad de la territorialización de la atención a la salud mental. Para ello, se propone responder a la siguiente pregunta: ¿cuáles son las características sociodemográficas del territorio ocupado por los niños, las mujeres y los adolescentes en el área de influencia del CAPSij Brasil? Metodología: Estudio seccional que permite analizar y evaluar los riesgos para la salud colectiva a los que está expuesta una población, especialmente los socioeconómicos y ambientales. La georreferenciación describe y permite la visualización de la distribución espacial, marcando los determinantes sociales y los factores etiológicos que pueden formular hipótesis y señalar asociaciones entre los fenómenos. Con la tecnología GIS buscamos delimitar el área de cobertura del servicio, así como clasificar lo que llamamos complejidades para la territorialización de la atención en salud mental. Resultados: La distribución espacial de las familias (mujeres, niños y adolescentes negros), acompañada del CAPSij muestra la segregación territorial que vive la población pobre y negra. Los territorios ocupados por la mayoría de la población negra son retratos del racismo que estructura el tejido social brasileño. Conclusión: los territorios son producto de las relaciones sociales, y pueden conferir ventajas y desventajas vinculadas a la ubicación, las infraestructuras, la movilidad, la educación, el trabajo, la salud, la cultura, etc. Esta realidad configura los procesos de salud mental de la población negra. Este estudio sobre la variación espacial del recorte de raza/color en los servicios de salud pública nos permite ver cómo las desigualdades raciales repercuten en el acceso al derecho a la salud, y señalar las intervenciones desde la perspectiva de los derechos humanos.

Palabras clave: Territorio; Salud mental; Población negra; Derechos.

\section{Introdução}

Os Centro de Atenção Psicossocial (CAPS) são os dispositivos estratégicos da Política Nacional de Saúde Mental, e compõem a Rede de Atenção Psicossocial (RAPS), serviços de saúde mental abertos, com o objetivo de acompanhar as pessoas com problemas de saúde mental, residentes em sua área de abrangência, por meio de acompanhamento clínico, e da reinserção social pelo acesso ao lazer, trabalho, exercício da cidadania, fortalecimento dos laços familiares e comunitários (Brasil, 2011).

O primeiro CAPS do Brasil foi criado na cidade de São Paulo em 1987, obtendo reconhecimento oficial como serviço de saúde com a publicação da Portaria GM n. ${ }^{\circ}$ 224/92, que os apresentava como serviços de cuidados intermediários entre o regime ambulatorial e a internação hospitalar. A implantação desse serviço, na perspectiva dos direitos sociais, ganhou forças a partir da Lei n. ${ }^{\circ}$ 10.216/2001 e da Portaria n. ${ }^{\circ}$ 336/2002. A Lei Federal n. ${ }^{\circ} 10.216$ reorientou o modelo assistencial no país ao dispor sobre a priorização do tratamento em serviços de base comunitária e, também, sobre a proteção e os direitos das pessoas com problemas de saúde mental, com a progressiva extinção dos manicômios como Política Nacional de Saúde Mental (Brasil, 2001).

O CAPS tem também como ação estratégica entender a complexidade do território para garantir o direito à saúde das populações, principalmente aquelas em situações de maior vulnerabilidade. As ações e intervenções dos serviços comunitários da Rede de Atenção Psicossocial (RAPS) devem ser pautadas na busca, defesa e promoção da cidadania de direitos, com implicações sobre os micro determinantes descritos por (Saraceno, 2020) como as condições de vidas das pessoas com problemas de saúde mental, a partir dos territórios onde habitam e vivem, do acesso à saúde, trabalho, alimentação, cultura, educação, saneamento, mobilidade; ter poder contratual, voz e voto, participação e controle social das políticas e práticas de saúde. 
$\mathrm{Na}$ afirmação e defesa da atenção psicossocial comunitária, em redes intersetoriais, e pela compreensão de que o cuidado deve ocorrer onde a vida acontece, é de fundamental importância para o campo psicossocial captar processos de discriminação e de inclusão/exclusão na infância e adolescência, o que pode fundamentar políticas, projetos e ações com vistas a reduzir mortalidade, problemas de saúde mental e internações de longa permanência em instituições asilares.

No Brasil, a maioria da população se autodeclara negra (51\%). Embora seja maioria demográfica, o grupo formado pelas pessoas negras, apresenta os piores indicadores sociais, além de ocupar no campo e nas cidades as áreas mais desprovidas de equipamentos e serviços públicos e privados. Em termos salariais, os negros (pretos e pardos) ganham $57 \%$ do salário dos brancos; 80\% das pessoas negras ganham até dois salários mínimos; brancos têm em média 9 anos de estudo contra 7,4 dos negros; a chance de pretos completar um curso universitário de engenharia é a metade da que tem os brancos, e no caso de odontologia é cinco vezes menor que a de um branco; $75 \%$ das pessoas que se declaram negras utilizam apenas os serviços públicos de saúde, em comparação com uma proporção de 50\% de pessoas brancas, os negros sofrem mais com a violência policial em comparação os brancos (Oxfam, 2017). Para os autores esse processo de invisibilidade e exclusão configuram o "racismo à brasileira".

No campo da Saúde, estudos evidenciam que 60,9\% dos usuários da Atenção Primária em Saúde são negros. Mesmo que seja maioria dos partos pelo Sistema Único de Saúde (SUS), as mulheres negras tiveram muito menos pré-natal, menos anestesia na hora do parto, menos orientações sobre aleitamento materno, e também tiveram menos acompanhantes no parto do que as mulheres brancas (Leal et al., 2005). Outros índices, tais como acesso a exames ginecológicos, dentistas, são também menores para as mulheres negras, o que configura como impacto do racismo à brasileira na saúde da população.

Nos últimos anos, houve um crescimento do corpo de evidências epidemiológicas que mostram fortes associações entre racismo e piores condições de saúde da população negra (Faro \& Pereira, 2011; Kwate \& Goodman, 2015). Observa-se, também, um aumento no interesse de pesquisadores em examinar o impacto da variável raça/cor, para a saúde e bem-estar de crianças e adolescentes, numa sociedade marcada por relações raciais estratificadas promotora de estereótipos, preconceitos e discriminações, como evidenciado na revisão sistemática (Priest et al., 2013).

A revisão narrativa da literatura (Silva et al., 2017) apresenta três artigos com dados sobre a presença do negro no perfil dos usuários atendidos em CAPS. Entretanto, "nenhum dos três artigos discutiu os achados, ou seja, raça/cor não foi considerada como categoria de análise da integralidade, universalidade e equidade do atendimento em saúde.

Barros, et al. (2020) ao estudar a atenção psicossocial a crianças e adolescente negros usuários do Centro de Atenção Psicossocial infantojuvenil (CAPSij) - Brasilândia, aponta a importância de se entender o território de responsabilidade do CAPS para adequar/construir Projetos Terapêuticos Individuais (PTS) e o Projeto Terapêutico Institucional (PTI), promovendo assim melhor qualidade no cuidado. Logo, mais que fazer a territorialização é fundamental que o serviço entenda a complexidade do território na perspectiva da atenção à saúde mental. As ações e serviços de saúde mental precisam integrar a perspectiva racial nas análises dos territórios onde estão inseridos os serviços da rede de atenção à saúde mental, para assim promover equidade em saúde.

Para Santos (p.14)

O território não é apenas um conjunto de sistemas naturais e de sistema de coisas superpostas. O território tem que ser entendido como o território usado, não o território em si. O território usado é o chão mais a identidade. A identidade é o sentimento de pertencer àquilo que nos pertence. O território é o fundamento do trabalho, o lugar da residência, das trocas materiais e espirituais e do exercício da vida (Santos, 2007). 
O território é um produto histórico de processos sociais e políticos que surge das condutas de territorialidades nele inseridas (Little, 2002). Condutas, porque territorialidades são manifestações e expressões de uso que equipam territórios, conferindo-lhe funcionalidade.

Além do uso do território e das manifestações materiais e imateriais de uso, portanto suas territorialidades, outra dinâmica territorial que tem relação com a distribuição espacial das pessoas é a de territorialização. De forma bem direta, definimos territorialização como o agir no território. A abertura de loteamentos residenciais, por exemplo, se configura em ação para territorialização de moradias pelo Estado ou setor imobiliário. Assim como a instalação de Unidade Básica de Saúde (UBS), Escolas, CAPS podem servir de exemplo para outro tipo de territorialização.

Nesse sentido, a ação política é também agente de delimitação territorial, do espaço geográfico, que a partir do uso que lhe for conferido passa a ser habitualmente denominado pela administração pública como território de atuação da gestão pública, ou de atendimento da política.

Esses territórios da ação política nem sempre abarcam o território de vida e vivência das pessoas, ainda que paradoxalmente sejam criados para atendê-las. Isso acontece porque em muitas ocasiões a ação criadora da política pública, se sobrepõe aos territórios de vida e vivência já existentes desconsiderando as dinâmicas das pessoas. E isso, por vezes, acaba por gerar conflitos, ou ainda a subutilização dos equipamentos e serviços (territorialidade governamental) instalados. A territorialidade governamental na maioria das vezes não segue a lógica da territorialidade no território, por desconhecer em muitos casos as identidades e pertencimento do grupo no território destinatário da política.

Diante disso, não podemos perder de vista que territorialidade é sempre uma estratégia, uma solução para quem as cria. E não um constrangimento, empecilho ou dificuldade. Logo, a territorialização de qualquer ação, programa e/ou política precisa considerar as territorialidades, que podem se apresentar na forma de especificidades culturais, demográficas, ambientais, sociais, econômicas e geográficas (físico-territoriais e socioterritoriais) da população destinatária da política pública. Aspectos, nem sempre observados no momento do planejamento e da implementação da política.

É nesse contexto de planejamento, monitoramento, avaliação e diagnóstico das políticas e serviços que o georreferenciamento se insere como uma ferramenta essencial e importante para a apreensão da distribuição das pessoas e serviços, assim como, das condições de acesso e uso desses serviços no território.

Este estudo pretende integrar análises geográficas, sociais e de saúde considerando as variáveis raça/cor, para compreensão da complexidade do cuidado territorializado em saúde mental e responder à seguinte questão: Quais as características sociodemográficas do território ocupado pelas crianças, mulheres e adolescentes na área de abrangência do CAPSij Brasilândia?

\section{Metodologia}

Para compreensão da realidade local e propiciar melhorias na qualidade da atenção à saúde mental de crianças e adolescentes, temos como desafio articular metodologias que permitam a apreensão da complexidade do território, considerando as variáveis geográficas, sociais e de saúde, com vistas a qualificar a territorialização do cuidado. Inicialmente adotamos para esta fase da pesquisa procedimentos embasados em estudos seccionais ou de corte transversais, isso porque este tipo de estudo quantitativo permite engendrar "investigações que produzem retratos da situação de saúde de uma população ou comunidade, sendo de grande utilidade para realização de diagnósticos comunitários da situação local de saúde" (Estrela, 2018).

Ainda como etapa metodológica utilizaremos a tecnologia SIG, buscando delimitar a área de abrangência do serviço, bem como, classificar aquilo que denominamos por complexidades do território para territorialização do cuidado em saúde mental. A aplicação do SIG na pesquisa em saúde oferece grandes possibilidades aos pesquisadores na aplicação de novos 
métodos para o manejo de sua informação espacial, tornando-se uma poderosa ferramenta para conexão entre saúde e ambiente.

O estudo da variação espacial produz um diagnóstico comparativo que pode ser utilizado das seguintes maneiras: indicar os riscos a que a população está exposta, acompanhar a disseminação dos agravos à saúde, fornecer subsídios para explicações causais, definir prioridades de intervenção e avaliar o impacto das intervenções. Assim, o georreferenciamento dos eventos de saúde é importante na análise e avaliação de riscos à saúde coletiva, particularmente as relacionadas com o meio ambiente e com o perfil socioeconômico da população descrever e permitir a visualização da distribuição espacial do evento; exploratório, sugerindo os determinantes locais do evento e fatores etiológicos a desconhecidos que possam ser formulados em termos de hipóteses e apontar associações entre os fenômenos.

\subsection{Cenário do estudo}

A área geográfica de estudo e de abrangência da Supervisão Técnica de Saúde da Freguesia/Brasilândia, é composta pelos distritos administrativos Freguesia do Ó e Brasilândia, localizados na Zona Norte do município de São Paulo/SP, com $21 \mathrm{~km}^{2}$ de referência do CAPSij Brasilândia. Com 280.069 habitantes segundo o IBGE (2010), o distrito encontra-se entre os quatro mais populosos da capital paulista.

\subsection{População}

A população do estudo compreende as crianças, adolescentes negros e as mulheres cuidadoras destas, acompanhados por um Centro de Atenção Psicossocial infantojuvenil (CAPSij) do município de São Paulo, referido ao ano de 2019. Em um primeiro momento, o CAPSij Brasilândia enviou à equipe de pesquisa uma planilha com a relação de 520 crianças e adolescentes usuários do CAPSij com prontuários ativos até o dia 29 de abril de 2019. Dos 387 prontuários ativos e com os dados sobre raça/cor, $186(48,1 \%)$ pessoas foram identificadas como brancas, 157 (40,6\%) como pardas, 40 (10,3\%) pretas, quatro (1\%) amarelas e nenhuma criança se autodeclarou indígena. Na apresentação dos resultados utilizaremos a categoria "negro" como referência à somatória de pretos e pardos, onde o total de crianças identificadas como negras igual a 197 $(50,9 \%)$.

\subsection{Coleta de dados}

As informações referentes ao ano de 2019 foram retiradas do campo endereço referido no prontuário da crianças/adolescente usuária do CAPSij e da Secretaria Municipal da Saúde de São Paulo. Utilizou-se o banco de dados que foi construído no Microsoft Excel 2010.

\subsection{Análise dos dados}

Para a obtenção do mapa temático, os dados foram geocodificados com auxílio do programa Arcap versão 2.6 e QGis versão 3.10.7. Utilizou-se as seguintes técnicas para composição dos mapas: método Kernel, mapa coroplético e georreferenciamento dos pontos (casos e serviços) pela localização do endereço de residência dos jovens.

Para a análise da declividade foi utilizado o Modelo Digital de Elevação proveniente da Plataforma Topodata do Instituto Nacional de Pesquisas Espaciais (INPE) (http://www.dsr.inpe.br/topodata/acesso.php). As classes de declividade em graus de inclinação do terreno foram estabelecidas em cinco níveis, de acordo com o modelo utilizado por Jin para a avaliação da adequação do uso do solo em regiões montanhosas de baixa declividade sob o impacto da urbanização em Yunnan, China (Jin et al., 2015). Para os autores consideram as melhores áreas aquelas com inclinações até $5^{\circ}$, entre $5^{\circ}$ e $8^{\circ}$ o terreno é moderadamente adequado, acima de $8^{\circ}$ é inadequado. Nessa fase, foram montados padrões de pontos de eventos da população 
do estudo. Os endereços referentes às notificações foram dispostos de forma a obter uma ligação com a criança, adolescente e mulher (mãe ou cuidadora) segundo endereço de residência na base cartográfica digital.

\subsection{Procedimentos éticos}

Para realização do referido estudo, o projeto foi previamente submetido à apreciação e aprovação pelo Comitê de Ética em Pesquisa da Escola de Enfermagem da Universidade de São Paulo, sob o CAAE: 68903417.7.0000.5392.

\section{Resultados}

A Figura 1 mostra que na área em que vive a população negra usuária do CAPSij se concentra maior quantidade de comunidades carentes, com maior vulnerabilidade social na garantia de direitos e acesso a bens e serviços. A história do distrito da Brasilândia é marcada pela política higienista do começo do século XX, pela expulsão de trabalhadores, principalmente, negros, moradores dos cortiços da região central de São Paulo. A Brasilândia está entre os sete distritos mais populosos e entre os 14 mais negros da cidade (RNSP, 2020).

Vale pontuar que o CAPSij está localizado na área nobre da supervisão de saúde Freguesia/Brasilândia, enquanto as crianças/jovens negras e suas cuidadoras residem nas regiões dos morros da Brasilândia. A proporção de domicílios em favelas é de 25,8\% na Brasilândia, e de 4,1\% na Freguesia do Ó, em relação ao total de domicílios, por distrito. A população jovem (0 a 29 anos) na Brasilândia é de 48,1\%, enquanto no distrito da Freguesia é 37,7\% (RNSP, 2020).

Figura 1. Localização dos Distritos de Brasilândia e Freguesia do Ó, São Paulo, SP e pontos da localização das moradias das crianças/adolescentes usuárias do CAPSij.

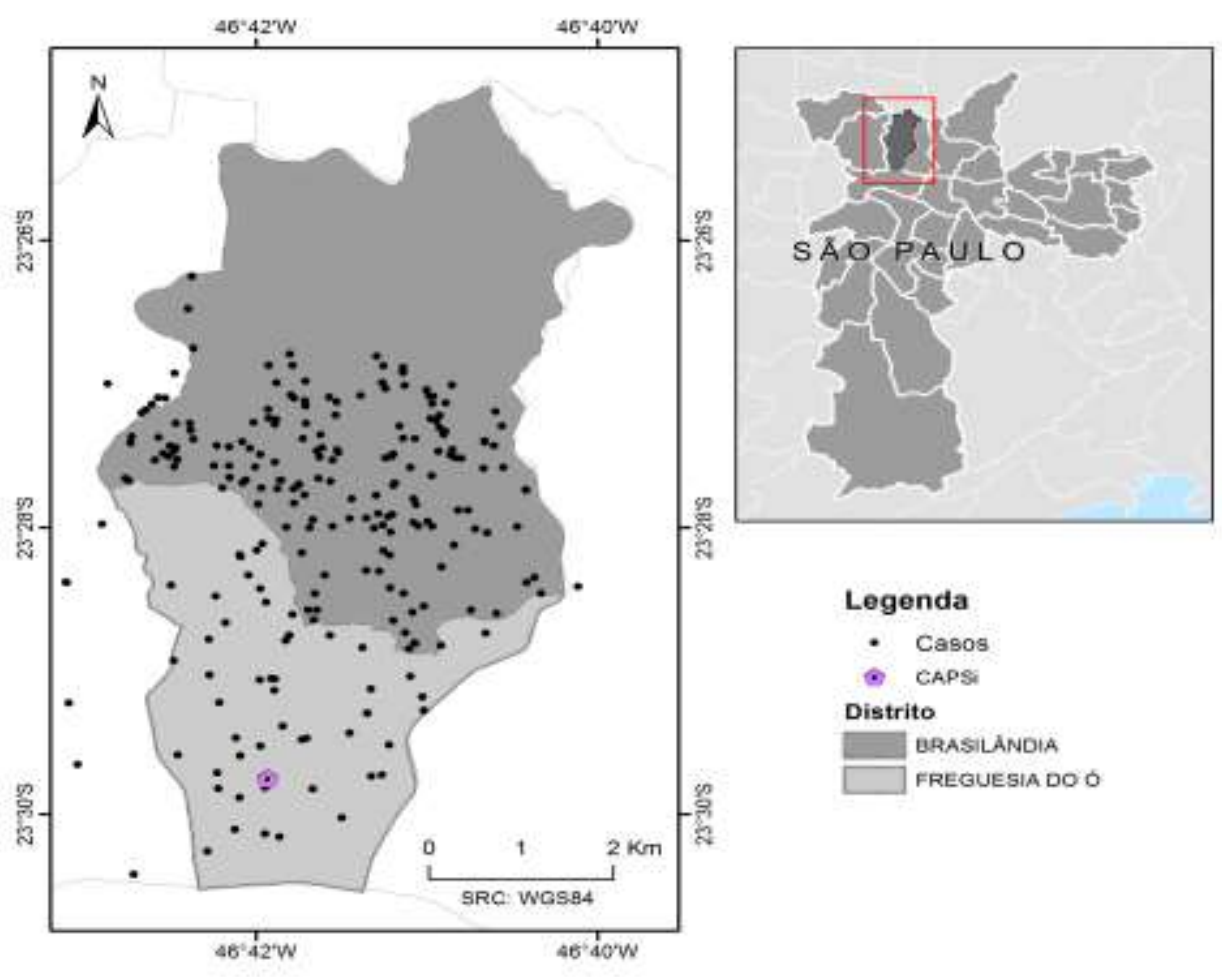

Fonte: Endereços que constavam nos prontuários das crianças e adolescentes. 
Os mapas abaixo são resultados do cruzamento da distribuição espacial dos endereços nos prontuários e área de abrangência do CAPSij em sobreposição a dados espaciais de altimetria, renda média da família (Figura 2a), população negra (Figura 2b) e mulheres chefe de família (Figura 2c), agrupados por setor censitário.

Figuras 2a, 2b e 2c. distribuição por renda, pop. negra e mulheres responsáveis pelo cuidado.
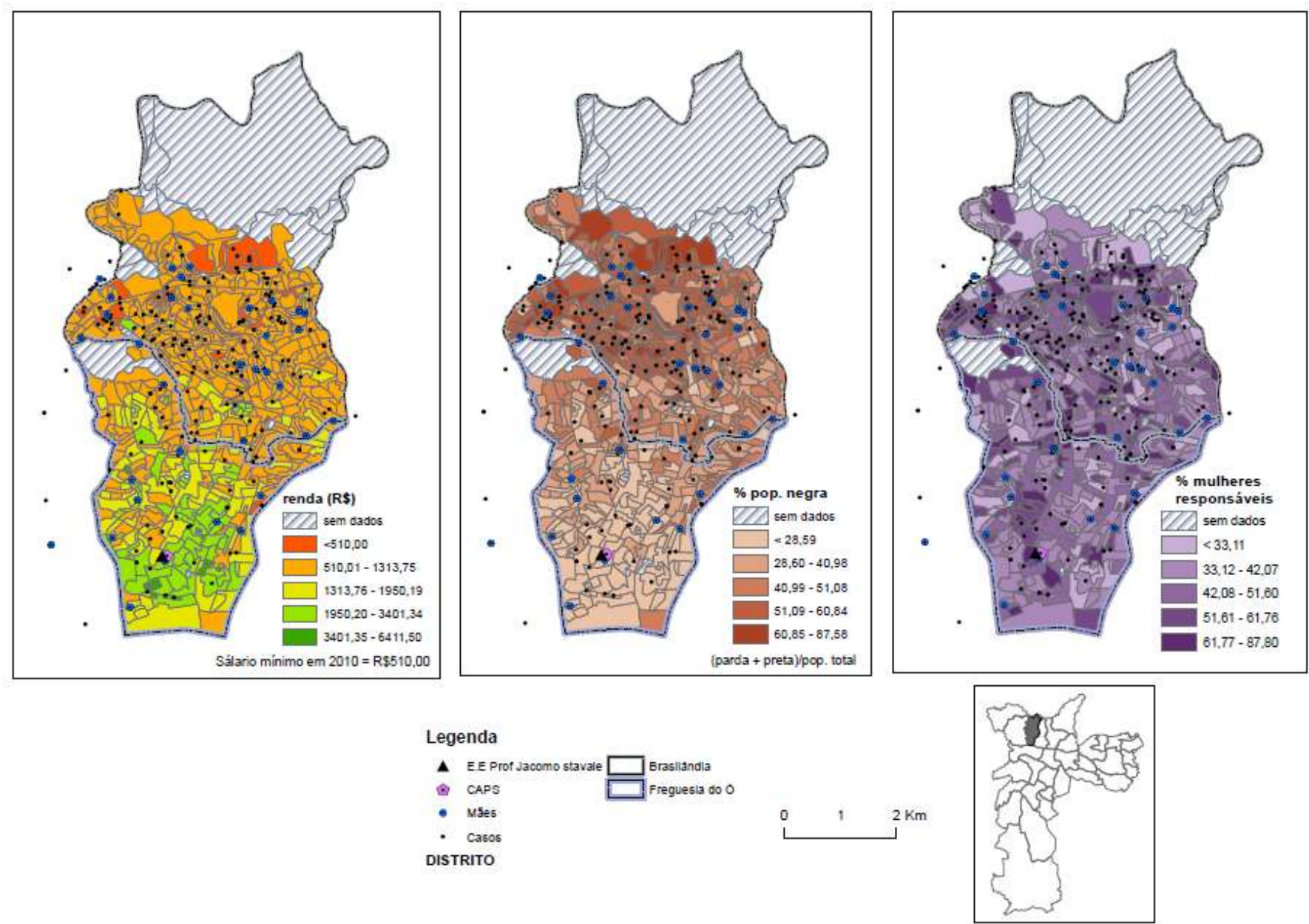

Fonte: Endereços que constavam nos prontuários das crianças e adolescentes.

A escolaridade e a renda per capita de chefes de família possui múltiplas implicações para o desenvolvimento das crianças e adolescentes, dificuldade no acesso a informações e serviços, limitações ao exercício da cidadania, interferindo diretamente nas condições de vida da população da região. O índice do nível socioeconômico das escolas no ano base 2015 na Brasilândia é 51,9, na Freguesia do Ó é 55,4, enquanto a média do município de São Paulo é de 56,0, indicador considera as escolas públicas e classifica questões sobre renda familiar, acesso a bens e serviços e nível de escolaridade de pais e responsáveis pelos estudantes. A evasão escolar no ensino fundamental da rede municipal, em proporção, no distrito da Brasilândia é de 1,5\%, na Freguesia do Ó é de 0,9\%.

No que diz respeito à mobilidade, quanto ao acesso de transporte em massa, na proporção da população que reside em até $1 \mathrm{~km}$ de estações e sistemas de transporte público de alta capacidade (trem, metrô e monotrilho) os distritos em discussão estão em $0 \%$, enquanto a média da cidade é $18,1 \%$. As famílias sem automóveis no distrito da Brasilândia equivalem a 59,6\% do total, na Freguesia do Ó 42,2\% das famílias não têm automóveis, a média da cidade é de 45,5\%. Quanto ao percentual da população que acessa a estrutura cicloviária no raio de 300 metros da residência, na Freguesia é de 70,8\%, na Brasilândia é de 
25,1\%. Ainda, o coeficiente de mortes em acidentes de trânsito para cada cem mil habitantes por distrito, na Brasilândia é de 4,6\% e na Freguesia do O é de 2,1\% (RNSP, 2020).

Abaixo, a Figura 3 demonstra que em áreas com maiores declividades, ou seja, onde o relevo é desfavorável à mobilidade e a urbanização, é onde se concentra a maioria de mulheres negras responsáveis pelo cuidado das famílias. Segundo o Mapa da Desigualdade 2020 a renda média familiar mensal no distrito da Brasilândia é R\$2.866,37, enquanto na Freguesia é R\$ 4.088,71 (RNSP, 2020).

Figura 3. Declividades do terreno em graus.

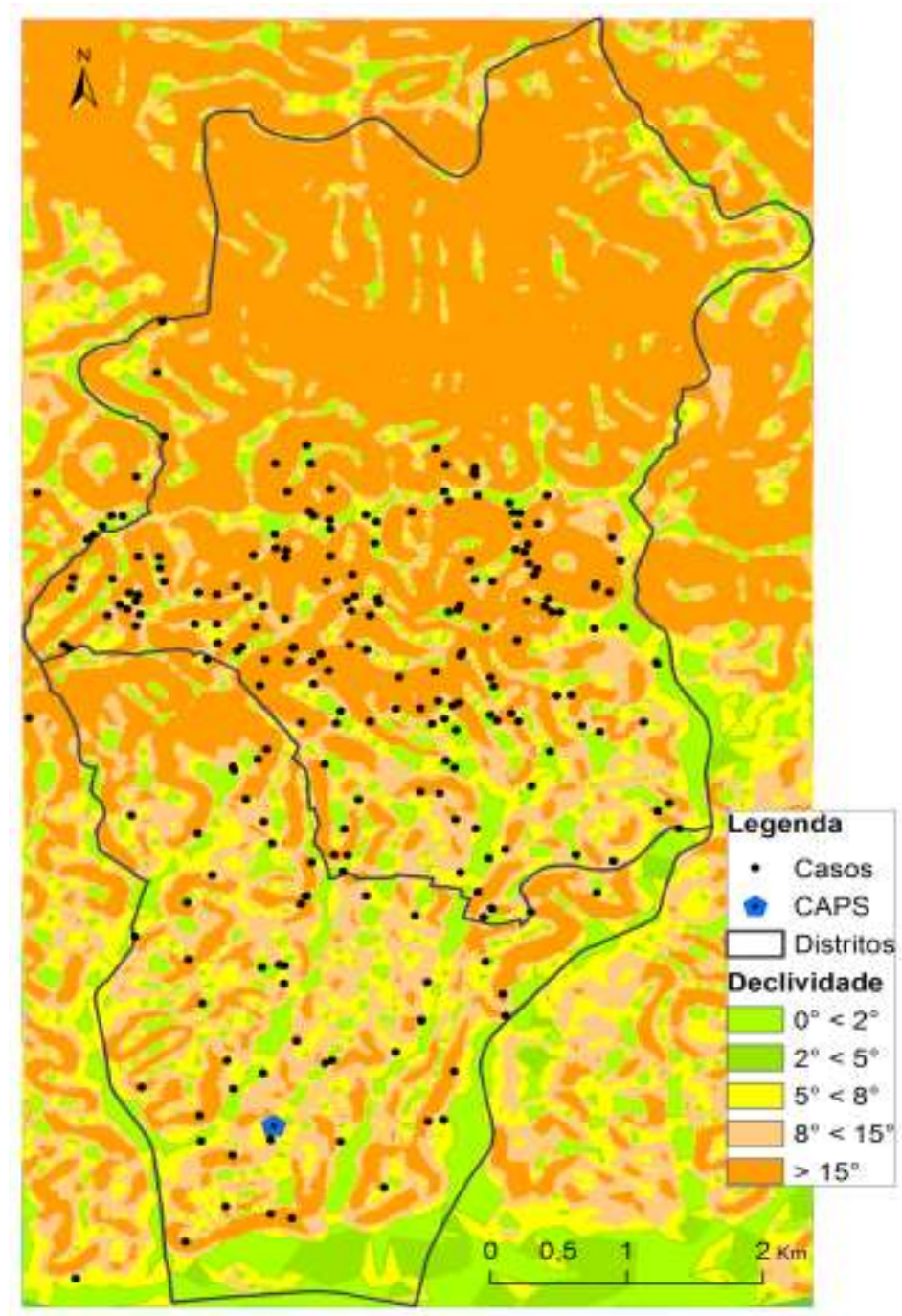

Fonte: Endereços que constavam nos prontuários das crianças e adolescentes.

A Figura 4 representa a concentração de casos e a localização dos serviços públicos, a distribuição das Unidades Básicas de Saúde, na relação com o local de moradia e a disponibilidade de equipamentos de saúde públicos no território de abrangência do CAPSij. Em relação ao mapa 4b, destaca-se a concentração dos casos em região periférica e com menos serviços especializados, mas as UBS estão presentes no território periférico, próximas às moradias da população negra, que representa $70 \%$ das(os) usuárias(os) do SUS. 
Figura 4. Distribuição dos serviços públicos na STS Freguesia do Ó/Brasilândia, 2020.
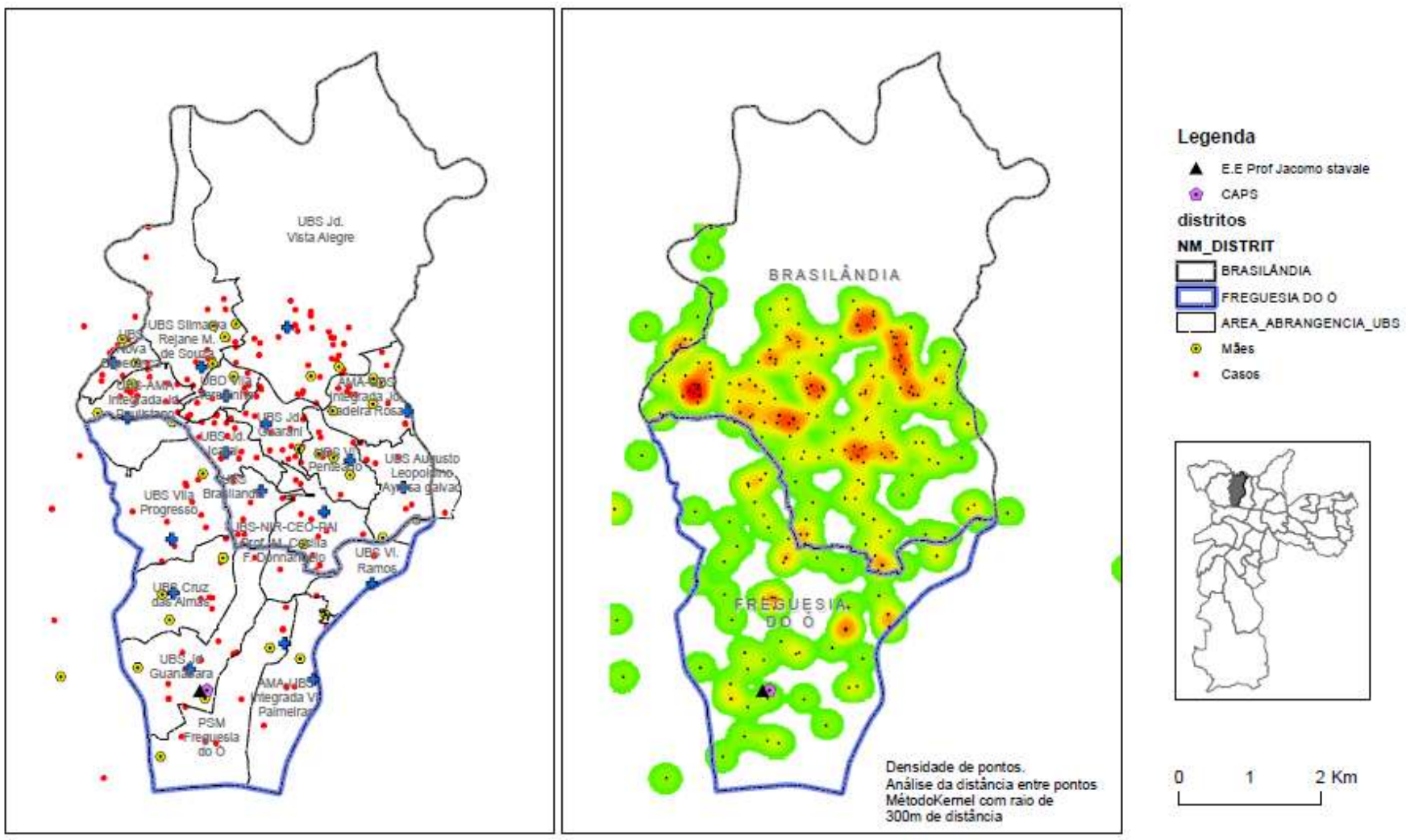

Fonte: Endereços dos serviços públicos segundo o site da Prefeitura de São Paulo.

Quanto a alguns dados sobre saúde da população nos distritos da Freguesia do Ó e Brasilândia a gravidez na adolescência, em proporção de nascidos vivos cujas mães tinham 19 anos ou menos é significativamente desigual: 7,6\% a 13,3\%. E a mortalidade infantil enquanto na Brasilândia é 18,9\%, na Freguesia do Ó é 10,6\%.

A idade média ao morrer, na Freguesia do Ó é 69,9 anos e na Brasilândia 60,5 anos. A Rede de Atenção Psicossocial da supervisão técnica de saúde dos dois distritos é composta por 40 serviços de saúde, 14 são UBS distribuídas por todos os bairros. Possui três equipes Núcleo Ampliado de Saúde da Família (NASF), segundo dados da prefeitura de 2015, as quais fazem o apoio matricial de 47 equipes de ESF nos casos com projetos terapêuticos singulares complexos. Com cobertura de 96,2\% de Equipes da Estratégia de saúde da família ou equipes de atenção básica na Brasilândia e 74,4\% na Freguesia (RNSP, 2020; São Paulo, 2021).

\section{Discussão}

Nas últimas três décadas crescem as análises sobre a espacialização dos grupos de pessoas por raça/cor com o propósito de verificar se a distribuição espacial das pessoas configura a racialização do espaço geográfico. Vale ressaltar que o uso e apropriação do espaço geográfico pelas pessoas, empresas e instituições públicas e privadas transformam o espaço geográfico em território. Santos define espaço geográfico como "fato e fator social”, também "como um conjunto indissociável de um sistema de ações e de um sistema de objetos" (Santos, 1994). Essas concepções de espaço geográfico permitem pensarmos a distribuição espacial das pessoas considerando as ações de saúde atreladas aos equipamentos disponíveis para realização e efetivação dessas mesmas ações, enquanto política pública e direito à saúde, bem como se dá o acesso e uso dos equipamentos e serviço de saúde. 
Em revisão sistemática da literatura (Arcaya et al., 2016), aponta o crescimento de pesquisas sobre efeitos do contexto de residência na saúde das populações, a segregação espacial racializada e o impacto na saúde amplia o terreno para estudos sobre a localização de causas de doenças e intervenções para melhorar a saúde coletiva (Krieger et al., 2017).

Nos diferentes e por vezes frágeis usos do conceito de território no campo da saúde mental, a organização do trabalho não ultrapassa a área de abrangência, ou ações fora do espaço físico das instituições, este estudo justifica a necessidade dos serviços operarem na lógica do território vivo, onde a vida acontece, nos laços e entrelaços relacionais, horizonte para o qual os serviços da RAPS devem olhar, escutar, atuar. Consideramos território de vida na perspectiva do espaço de vida de Di Méo (Koga, 2003), "como aquele onde se desenvolvem práticas cotidianas dos sujeitos em torno do seu local de moradia, trabalho, como também de suas práticas episódicas, como lugares de lazer, férias, etc.” E território de vivências, como aquele que é "ilimitado, reconstruído mentalmente pelos sujeitos ou representado pelo seu imaginário".

Neste sentido, os profissionais de saúde mental devem explorar o território com a finalidade de expandir e integrar a RAPS com os demais dispositivos sociais e construir parcerias por meio de ações que visem o enfrentamento do estigma social, do racismo e na perspectiva da inclusão social das crianças, adolescentes e das mulheres, mães, cuidadoras. Por outro lado, vivenciar o território onde o serviço de saúde mental está inserido contribui para o fortalecimento da estrutura da RAPS e na construção de ações que convergem com a autonomia, aumento da contratualidade, acesso e efetivação de direitos.

O CAPSij Brasilândia desenvolve ações de enfrentamento ao racismo, no entanto, a implementação da política de saúde mental numa perspectiva racial demandará considerar as especificidades culturais, demográficas, sociais, econômicas, ambientais e geográficas (físico-territoriais e socioterritoriais) da população destinatária da política pública. Os dados evidenciam que as famílias negras ocupam o espaço periférico, com menor poder de acesso a serviços no distrito da Brasilândia, demonstra-se com estes, a história de segregação racial e socioeconômica da constituição deste território na cidade de São Paulo. Fator contextual na causalidade de disparidades no acesso ao CAPSij Brasilândia.

O racismo é determinante social de saúde, violência que acarreta efeitos psicossociais e sofrimento, bem como vulnerabilidades psicossociais. O racismo cria barreiras de acesso a direitos e atinge as subjetividades de distintas formas, entender como o racismo atravessa a compreensão do mundo, assim como o racismo atravessa os territórios de vida das pessoas, é fundamental para enfrentar e transformar a crença da democracia racial, que tem reforçado obstáculos.

O CAPSij localizado na Freguesia do Ó, na compreensão do território de referência, e na perspectiva da equidade, precisa estender sua atuação no distrito da Brasilândia, aprimorar a organização do processo de trabalho transdisciplinar na perspectiva da territorialidade, na articulação entre os serviços, associações, movimentos sociais, culturais e outros equipamentos públicos e organizações da sociedade civil, com as necessidades de saúde das pessoas, viver o território para além dos muros institucionais permitirá ao CAPSij desenhar melhor a atenção aos usuários e aprimorar suas ações de enfrentamento ao racismo.

Esta questão aponta para a discussão proposta de Benedeto Saraceno sobre como os serviços de saúde mental podem agir a partir de micro intervenções no território. Para o autor, é necessário que os serviços se atentem para a complexidade da vida das pessoas e agirem considerando isso, esses desafios na saúde mental precisam ser enfrentados para as ampliar as práticas presentes e futuras (Saraceno, 2020).

Neste caminho, o estudo aponta a necessidade do CAPSij estar atento ao território para construir Projetos Terapêuticos Singulares (PTS) e Projeto Técnico Institucional (PTI). Para enfrentar e combater o racismo nos processos de cuidar em liberdade, em parceria com as Unidades Básicas de Saúde e Equipes de Estratégias da Família, reconhecendo a capilaridade e potência desses equipamentos enquanto estruturais no Sistema Único de Saúde, no trabalho compartilhado, construído no plural, gerido no coletivo pelos conselhos gestores, e como recomendam Lima e Yasui "estabelecendo alianças 
com grupos e movimentos de arte ou com cooperativas de trabalho, para potencializar as ações de afirmação das singularidades e de participação social" (Lima et al., 2014).

Ir de encontro ao território é estar atento aos modos de organização, de articulação, de resistência e sobrevivência das pessoas que ocupam esses espaços, como são suas casas, trabalhos e rendas, alimentação, rede social de suporte, acesso aos serviços e recursos do entorno. Os serviços estratégicos da RAPS tem por responsabilidade produzir, fortalecer, criar, ocupar o território com a loucura, "ao espaço público como locus terapêutico, de intervenção, de montagens e instalações em permanente processo de produção" (Lima et al., 2014).

O trabalho em rede e territorial tem implicações políticas e sociais, o espaço social em que o campo da Saúde Mental opera não é neutro, há aspectos econômicos, políticos, clínicos, morais, coloniais, que oferecem resistência à inserção social de pessoas negras com problemas de saúde mental. Os autores recomendam uma prática clínica delicada e atenta, capaz de acolher a multiplicidade de formas de existência e redes de sentido que criem novos territórios (Lima et al., 2014).

À luz do enfoque da Determinação Social da Saúde, torna-se fundamental a análise das condições macro e micro dos determinantes que constituem a vida das pessoas em um determinado território. O território é componente indissociável dos processos sociais e não apenas um terreno em que estes se desenvolvem, assim, as condições de vida e, consequentemente, de saúde, estão fortemente relacionadas aos espaços utilizados pelas pessoas, onde circulam, habitam, crescem, vivem, trabalham, envelhecem (Dimenstein et al., 2018).

Considerando que a promoção da equidade é um dos princípios do SUS é de responsabilidade do Estado a construção de ações que reduzam as iniquidades e injustiças sociais. Nesse sentido, a substituição do modelo de atenção, pautada na reestruturação da rede assistencial de base territorial e comunitária, mantém-se como desafio cotidiano para os gestores e trabalhadores da saúde mental. Uma sociedade sem manicômios passa pelo enfrentamento às desigualdades de classe, ao racismo, ao machismo, a homofobia, e a todas as formas de discriminação e preconceito. Neste terreno, agravada pelo momento de crise política, sanitária, econômica, e social, potencializada pela necropolítica instaurada pelo governo que é responsável pelo grande impacto da pandemia COVID-19 nas populações em situação de vulnerabilidade, articular as pautas dos movimentos sociais na luta pelos direitos pode fortalecer a Reforma Psiquiátrica Antimanicomial (Santos \&Vieira, 2018).

Conforme apontado, o uso do espaço geográfico o transforma em território, espaço de exercício de poder, também de resistência, aponta-se neste estudo a necessidade de pesquisas sobre a complexidade do território na perspectiva da atenção à saúde mental, que possibilite integrar análises, geográficas, raciais, sociais e de saúde para propiciar melhorias na qualidade da atenção à saúde mental, e aprimorar o controle e a participação social na Política de Saúde Mental.

\section{Considerações Finais}

A distribuição espacial das famílias (mulheres, crianças e adolescentes negros), acompanhadas pelo CAPSij mostra a segregação territorial vivenciado pela população pobre e negra. Os territórios ocupados pela maioria da população negra são retratos do racismo que estrutura a trama social brasileira. Para Santos (2007), os territórios são resultantes de processos históricos de uso social da vida, portanto, produto das relações sociais, que se manifesta como realidade objetiva das pessoas podendo conferir vantagens e desvantagens ligadas a localização, infraestrutura, mobilidade, educação, trabalho, saúde, serviços etc. Essa realidade configura os processos de saúde mental da população negra.

No aprofundamento, prático, teórico e político da territorialização do cuidado em saúde mental é preciso fortalecer e instituir redes, com abordagem transdisciplinar e intersetorial nos espaços de discussão e no acompanhamento das pessoas com problemas de saúde mental. Fato é que a reforma psiquiátrica brasileira antimanicomial sempre esteve permeada por represálias, enfrentamentos e oposições, para a transformação das relações sociais de discriminação e exclusão das pessoas 
com problemas de saúde mental, é necessário avanços nas práticas, no nível singular, na micropolítica do cotidiano, na participação e controle social, na contratualidade, na representação social da loucura, e na garantia de direitos.

Associado a dinâmicas de territorialização podemos identificar pelo menos três sujeitos: o Estado e organizações políticas; pessoas residentes; e empresas. Respectivamente esses sujeitos respondem: pelo controle e regulação da política; relações de identidade e de identificação; relações econômicas e trabalho. O CAPSij é articulador do cuidado em liberdade de crianças e adolescentes negros, e no que tange às micro intervenções de um CAPSij para produzir ações de enfrentamento ao racismo, se faz necessário incorporar como diretriz que o quesito "raça/cor" seja um dado analítico nos serviços de saúde mental incorporado ao PTI e ao PTS e incidir no controle social nas políticas públicas de saúde mental para crianças e adolescentes.

Estudos sobre a variação espacial de serviços públicos e o espaço ocupado pelas crianças, adolescentes usuários do CAPSij e sua família, possibilitam visibilizar como as desigualdades raciais impactam no acesso ao direito à saúde, e intervir na perspectiva dos direitos humanos. Nesse sentido, os estudos futuros sobre a variação espacial de serviços públicos na relação com o espaço ocupado pela população negra, maioria de pessoas usuárias do SUS, poderão desvelar como as desigualdades raciais impactam no acesso ao direito à saúde no território de abrangência georreferenciada, a fim de subsidiar equitativamente as práticas e políticas de saúde na perspectiva dos direitos humanos nos lugares com desigualdade social e racial.

\section{Agradecimentos}

Fundação de Amparo à Pesquisa do Estado de São Paulo (FAPESP), processo nº 2018/07251-2.

\section{Referências}

Arcaya, M. C. et al. (2016). Research on neighborhood effects on health in the United States: A systematic review of study characteristics. Social Science \& Medicine, 168,16-29.

Barros, S., Batista, L. E., Santos, J. C., Messias, L., Martins, L. R., Ballan, C., et al. (2020). Relatório técnico do diálogo deliberativo “O processo de cuidar em saúde mental da criança/ adolescente negro usuário de CAPSij”. EEUSP.

Brasil. Lei n. ${ }^{\circ} 10.216$ de 06 de abril de 2001. (2001). Dispõe sobre a proteção e os direitos das pessoas portadoras de transtornos mentais e redireciona o modelo assistencial em saúde mental. Diário Oficial da União. Presidência da República.

Brasil. Portaria n. 336 de 19 de fevereiro de 2002. (2002). Estabelece que os Centros de Atenção Psicossocial poderão constituir-se nas seguintes modalidades de serviços: CAPS I, CAPS II e CAPS III, definidos por ordem crescente de porte/complexidade e abrangência populacional. Diário Oficial da União. Brasília, DF: Ministério da Saúde.

Brasil. Portaria n. 3.088 de 23 de dezembro de 2011. (2011). Institui a Rede de Atenção Psicossocial para pessoas com sofrimento ou transtorno mental e com necessidades decorrentes do uso de crack, álcool e outras drogas, no âmbito do Sistema Único de Saúde (SUS). Diário Oficial da União. Brasília, DF: Ministério da Saúde.

Dimenstein, M., Siqueira K., Macedo J. P., Leite J. \& Dantas C. (2017). Determinação social da saúde mental: contribuições à psicologia no cuidado territorial. Arquivos Brasileiros de Psicologia, 69(2), 72-87.

Faro, A. \& Pereira, M. E. (2011). Raça, racismo e saúde: a desigualdade social da distribuição do estresse. Estudos de Psicologia, 16(3), 271-8.

Freire, M. C. M., \& Pattussi M. P. (2018). Tipos de estudos. In Estrela, C. (2018). Metodologia Científica: Ciência, Ensino, Pesquisa (pp.109-127). Editora Artes Médicas.

Harris, R., Cormack, D., Tobias, M., Yeh, L.-C., Talamaivao, N., Minster, J., \& Timutimu, R. (2012). The pervasive effects of racism: experiences of racial discrimination in New Zealand over time and associations with multiple health domains. Social Science \& Medicine, 74(3), 408-15.

IBGE. (2019). Pesquisa Nacional por Amostra de Domicílios Contínua 2016-2018 (livro 101657). IBGE, Diretoria de Pesquisas, Coordenação de Trabalho e Rendimento.

Jin, G. et al. (2015). Land Use Suitability Assessment in Low-Slope Hilly Regions under the Impact of Urbanization in Yunnan, China. Advances in Meteorology, 2015.

Koga, D. (2003). Medidas de cidades. Cortez. 
Research, Society and Development, v. 10, n. 10, e215101018848, 2021

(CC BY 4.0) | ISSN 2525-3409 | DOI: http://dx.doi.org/10.33448/rsd-v10i10.18848

Krieger, N. et al. (2017). Local Residential Segregation Matters: Stronger Association of Census Tract Compared to Conventional City-Level Measures with Fatal and Non-Fatal Assaults (Total and Firearm Related), Using the Index of Concentration at the Extremes (ICE) for Racial, Economic, and Racialized Economic Segregation, Massachusetts (US), 1995-2010. Journal of Urban Health: Bulletin of the New York Academy of Medicine, 94(2), 244-58.

Kwate, N. O. A. \& Goodman, M. S. (2015). Racism at the intersections: Gender and socioeconomic differences in the experience of racism among African Americans. The American Journal of Orthopsychiatry, 85(5), 397-408.

Leal, M. do C., Gama, S. G. N. da \& Cunha, C. B. da. (2005). Desigualdades raciais, sociodemográficas e na assistência ao pré-natal e ao parto, $1999-2001$. Revista de Saúde Pública, 39(1), 100-7.

Lima, E. M. F. de A., de Araújo Lima, E. M. F. \& Yasui, S. (2014). Territórios e sentidos: espaço, cultura, subjetividade e cuidado na atenção psicossocial. Saúde em Debate, 38(102).

Little, P. E. (2018). Territórios sociais e povos tradicionais no Brasil: por uma antropologia da territorialidade. Anuário Antropológico, 28(1), 251-90.

Oxfam. (2017). A distância que nos une: um retrato das desigualdades brasileiras. Oxfam Brasil.

Priest, N. et al. (2013). A systematic review of studies examining the relationship between reported racism and health and wellbeing for children and young people. Social Science \& Medicine, 95, 115-127.

RNSP. (2020). Mapa da Desigualdade 2020. Rede Nossa São Paulo. https://www.nossasaopaulo.org.br/wp-content/uploads/2020/10/Mapa-da-Desigualdade2020-TABELAS-1.pdf

Santos, M. (1994). Técnica, espaço, tempo: globalização e meio técnico-científico informacional. EDUSP.

Santos, M. (2007). Espaço do Cidadão. (7a ed.), Editora da USP.

Santos, I. S. \& Vieira F. (2018) Direito à saúde e austeridade fiscal: o caso brasileiro em perspectiva internacional. Ciência \& Saúde Coletiva, 23(7), 2303-14.

São Paulo, S. P. (2021). Relação dos Estabelecimentos/Serviços da Secretaria Municipal da Saúde por Coordenadoria Regional de Saúde e Supervisão Técnica de Saúde. https://www.prefeitura.sp.gov.br/cidade/secretarias/upload/saude/arquivos/organizacao/Unid_Munic_Saude_Super.pdf

Saraceno, B. (2020). O futuro da psiquiatria e da saúde mental. Saúde Em Debate, 44(Esp. 3), 29-32.

Silva, N. G., Barros, S., de Azevedo, F. C., Batista, L. E. \& Policarpo, V. C. (2017). O quesito raça/cor nos estudos de caracterização de usuários de Centro de Atenção Psicossocial. Saúde e Sociedade, 26(1), 100-14). 